\title{
Hall and Pearce negative transfer: Assessments in conditioned suppression and nictitating membrane conditioning experiments
}

\author{
JOHN J. B. AYRES, JOHN W. MOORE, and MICHAEL VIGORITO \\ University of Massachusetts, Amherst, Massachusetts
}

\begin{abstract}
Two experiments using a conditioned suppression procedure in the rat replicated the finding of Hall and Pearce (1979) that pairing a conditioned stimulus (CS) with a weak unconditioned stimulus (US) in Stage 1 produces negative transfer when US intensity is increased in Stage 2. Although the degree of negative transfer appeared to be independent of initial CS salience, it did depend on other factors. Specifically, weak suppression in Stage 1, or a postasymptotic decline in suppression in Stage 1, appeared crucial: strong suppression in Stage 1 unaccompanied by a postasymptotic decline resulted in positive, not negative, transfer. Experiments using the nictitating membrane response (NMR) conditioning procedure in the rabbit and a wide range of parameters failed to find evidence for negative transfer, but as a rule showed positive transfer instead. Because Kamin blocking, conditioned inhibition, and negatively accelerated learning curves are all readily obtained with the rabbit NMR procedure, the strong possibility exists that the mechanisms responsible for these effects differ from those responsible for the Hall-Pearce negative transfer phenomenon.
\end{abstract}

Several theories of associative learning formulated in the last decade are based on the idea that selective associations during classical conditioning are a result of changes in the effectiveness of a target conditioned stimulus (CS) for entering into association with the unconditioned stimulus (US). Some of these theories ascribe selective associations to changes in the effectiveness of the US (e.g., Rescorla \& Wagner, 1972; Schull, 1979; Wagner, 1976, 1978; other theories ascribe selective associations to changes in the effectiveness of the target CS (e.g., Dickinson \& Mackintosh, 1979; Mackintosh, 1975; Moore \& Stickney, 1980).

The Rescorla-Wagner model, for example, states that the amount of learning that occurs on any given trial depends on the discrepancy between the associative asymptote supportable by that US and the total associative strength of all CSs on that trial: When the discrepancy is very small or zero, the US cannot become associated with any newly introduced stimulus with which it is paired. Wagner's theory (Wagner, 1976, 1978) described a mechanism by which the US loses effectiveness as its representation is increas-

Support for this research was provided by National Science Foundation Grant BNS 8100322 . The authors are grateful for the assistance of Hayley Arnett and Bonnie Dyer-Bennett, who carried out the experiments described in Part B, and Chuck Peck, who helped conduct Experiment 2A. We also wish to thank Geoffrey Hall and John Pearce for extensive discussion about their model and for comments on a previous draft of the manuscript. Requests for reprints should be directed to any of the authors at the following address: Department of Psychology, Middlesex House, University of Massachusetts, Amherst, MA 01003. The order of authorship was randomly determined; all three authors contributed equally. ingly better primed into short-term memory by CS presentations.

Theories that appeal to changes in the effectiveness of a target CS assume that it is the associability of the CS that changes during conditioning. Typically, such models assume that CS-US pairings increase CS associability (e.g., Mackintosh, 1975; Moore \& Stickney, 1980) or at least maintain it (Lubow, Weiner, \& Schnur, 1981). In such models, as in the Rescorla-Wagner model, associability is one of the parameters controlling the rate of learning. Recently, however, Pearce and Hall (1980) presented a model that assumes that CS-US pairings decrease CS associability.

According to this model, when a single CS (A) is reinforced on trial $\mathrm{n}$, the change in its associative strength is a product of three factors: the intensity of the CS $\left(\mathrm{S}_{\mathrm{A}}\right)$, the associability of the CS $\left(\alpha_{\mathrm{A}}\right)$, and the asymptotic level of conditioning $\left(\lambda^{n}\right)$ that the US on trial $n$ will support. This asymptotic level is, in turn, determined by the intensity of the US used on trial n, and each of the three factors is permitted to vary between 0 and 1 . An assumption unique to the model is that all CSs have associability initially, but lose it as they acquire associative strength or come to "fully predict their consequences" (Pearce \& Hall, 1980). Specifically, the associability of the CS $\left(\alpha_{\mathrm{A}}\right)$ is said to be proportional to the absolute value of the difference between $\lambda^{n-1}$ (i.e., $\lambda$ on trial $n-1$ ) and $V_{A}^{n-1}$ (i.e., the total associative strength acquired by Stimulus $A$ by the end of trial $n-1$ ).

This unique assumption of the model permits it to deduce a variety of Pavlovian conditioning phenomena. Among these is the seemingly paradoxical finding that 
pairing a CS with a weak US can retard conditioning when the CS is subsequently paired with a strong US. The model anticipates this result as follows: If (say for Group A) Stimulus $\mathrm{A}$ has been conditioned to asymptote with a weak US, then $\left|\lambda_{n-1}-V_{A}^{n-1}\right|$, or $\alpha_{A}$, should be essentially zero. Because of this, a large increase in US intensity on trial $\mathrm{n}$ could have no effect on that trial: no matter how intense the US was on trial $\mathrm{n}$, no increment in conditioned strength could occur, because the product of the three factors mentioned above would be zero. In contrast, if (for Group B) Stimulus A was novel on trial $\mathrm{n}$, then its associability would exceed zero, and so it would gain associative strength. That gain, moreover, being a fraction of $\lambda^{n}$, would increase directly with the trial $n$ US intensity. If that intensity was great enough, then Stimulus A would gain more associative strength on trial $\mathbf{n}$ for Group B than it gained in Group A on all of the preceding trials with the weak US.

In confirmation of this prediction, Hall and Pearce (1979), using a two-stage transfer-of-training design, showed that the acquisition of the conditioned suppression produced by pairing a CS with a strong US in Stage 2 was retarded if the CS had first been paired with a weak US in Stage 1. Pearce and Hall (1980) interpreted this negative transfer effect as supporting their hypothesis that CSs lose associability as $V_{A}$ approaches $\lambda$.

We should like to note, however, that the Pearce-Hall model does not always predict negative transfer. Suppose, for example, that the Stage $1 \lambda$ and the Stage $2 \lambda$ were very similar. On the first trial of Stage 2, Simulus A would gain associative strength in Group B, the group for which the CS was novel and $\alpha_{\mathrm{A}}$ presumed high; but that gain would be only a fraction of the Stage $2 \lambda$. In contrast, for Group A, the group with prior pairings of the CS and weak US, associative strength would already approximate the Stage $2 \lambda$. Thus, even though $\alpha_{\mathrm{A}}$ might be zero for Group $A$, and even though no gain in associative strength could occur on the first Stage 2 trial, Group A must show positive transfer relative to $B$, because Group $A$ has already reached an asymptote appropriate to Stage 2 .

The present experiments sought to replicate the HallPearce negative transfer effect. The first two used rats in the conditioned suppression procedure, as did Hall and Pearce (1979); the last experiments used rabbits in the conditioned nictitating membrane response (NMR) procedure (Gormezano, 1966). The two sets of experiments were initiated at the same time and pursued independently, using standard procedures appropriate to each. The first set found evidence for both negative and positive transfer; the second set found only positive transfer.

\section{PART A: CONDITIONED SUPPRESSION}

\section{Experiment 1A}

The conditioning procedures of Experiments $1 \mathrm{~A}$ and $2 \mathrm{~A}$ followed closely those used in the demonstration of the negative transfer effect described by Hall and Pearce (1979). The subjects were food-deprived rats, and they received auditory or visual CSs paired with weak or strong electric shock USs while barpressing for a food reward. Suppression of the barpress rate during conditioning trials was taken as an index of the strength of conditioning.

The purpose of Experiment $1 \mathrm{~A}$ was to study the effects of CS salience on transfer. Before performing this study, we conducted a pilot experiment to determine the relative salience of several CSs. Specifically, we compared the rate of acquisition of conditioned suppression to a tone CS with the rate produced by several visual CSs, including a flashing light CS. The US in this pilot experiment was a weak (.4-mA, .5-sec) scrambled-grid shock. The pilot study showed the light CS to be more salient than the tone. That is, the tone produced virtually no suppression of barpressing during 11 training days, whereas suppression to the light was quickly acquired. By the 2nd day of training, for example, responding to the light was about four times lower than the baseline rate. The pilot study also included a second stage in which all groups received conditioning to the tone but with a stronger $(.8-\mathrm{mA}, 1-\mathrm{sec})$ shock as the reinforcer. In this stage, we found slower acquisition of suppression to the tone in the group for which tone was the CS used in Stage 1 than in the groups for which light was the CS in Stage 1. This result thus replicated the negative transfer effect found by Hall and Pearce (1979) - the effect that led them to conclude that the target CS had lost associability as a result of its conditioning in Stage 1.

The purpose of the present experiment was to compare directly the amount of negative transfer obtained when the (nonsalient) tone was used as the target CS in Stage 2 (as in our pilot work) with that produced when the (more salient) light was used as the target CS. Since the light was known to produce moderate levels of suppression in Stage 1, there might be reason to expect positive transfer rather than negative transfer when the light served as the target CS.

\footnotetext{
Method

Subjects. Thirty-two male albino rats, 90 days old when obtained from the Holtzman Company, Madison, Wisconsin, were individually housed in a continuously lighted room and were kept at $80 \%$ of their free-feeding body weights.

Apparatus. Eight Gerbrands Skinner boxes were housed in ventilated $.61-\mathrm{m}$ cubes of $12.7-\mathrm{mm}$ plywood lined with acoustical tile. The inside dimensions of each chamber were $23.2 \times 20.3 \times 19.5 \mathrm{~cm}$. Each floor was composed of 18 stainless steel rods, $2 \mathrm{~mm}$ in diameter, mounted $1.3 \mathrm{~cm}$ apart center to center. The end walls were aluminum; the side walls and top were transparent Plexiglas. Centered in one end wall was a standard Gerbrands bar, $1.5 \times 5.0 \mathrm{~cm}$, mounted $8 \mathrm{~cm}$ above the grid floor. In the lower left corner of this same wall was a $5.5 \times 5.0 \times 5.0 \mathrm{~cm}$ dipper receptacle.

On the lid of each box were two $10-\mathrm{cm}$ speakers. One provided a nonintermittent $1000-\mathrm{Hz}$ tone $(\mathrm{T}) \mathrm{CS}$ at $84 \mathrm{~dB}$ intensity re $20 \mu \mathrm{N} / \mathrm{m}^{2}$; the other was not used in Experiment 1A; however, in Experiment $2 \mathrm{~A}$ it provided an intermittent ( $1 \mathrm{sec}$ on, .II sec off) white noise CS (N) of $80 \mathrm{~dB}$ intensity re $20 \mu \mathrm{N} / \mathrm{m}^{2}$. Also on the lid of the box was a 28-V lamp. A second 28-V lamp (a cue light) was mounted over the dipper opening $95 \mathrm{~mm}$ above the grid floor. A visual CS (L) was created by flashing the $28-\mathrm{V}$ cue lamp and roof lamp in synchrony (1 sec on, .11 sec off)
} 
against a normally dark background. This visual CS, created by flashing the two lights together, will be referred to as "the light CS." The tone and light used in this experiment were identical to the tone and light used in the previously mentioned pilot study.

Scrambled grid shocks served as USs and were provided by eight Grason-Stadler shock sources (Models E1064GS and 700). Barpressing, the baseline response to be suppressed by CS trials, was reinforced with $4-\mathrm{sec}$ presentations of a $.1-\mathrm{ml}$ dipper cup containing a $32 \%$ (by weight) sucrose solution.

Procedure. Preliminary training: Preliminary barpress training began with three daily sessions of barpress shaping and ended with five daily 1-h sessions under a VI 1-min schedule of reinforcement.

Stage 1: The 32 rats were assigned randomly to four groups: L-L, T-L, T-T, and L-T. Eight rats were assigned to each group. The groups were matched in terms of body weight and responding during preliminary VI training. Conditioning chambers were balanced across groups. In the group designation, the letter before the hyphen denotes the stimulus that was paired with a weak shock in Stage 1; the letter after the hyphen denotes the stimulus that was paired with a stronger shock in Stage 2.

Sessions in Stage 1 were $1 \mathrm{~h}$ long, and for groups that received conditioning, each session contained six 90 -sec CS trials that terminated in the onset of a $4-\mathrm{mA} .5$-sec scrambled grid shock US. Following Hall and Pearce (1979), the first trial began $7.5 \mathrm{~min}$ after the start of the session, and 10 min elapsed between the onsets of successive trials. There were 11 sessions in Stage 1.

Stage 2: Stage 2 was a search for evidence that the associability of a CS could be reduced as a result of being paired with weak shock in Stage 1 . Such a loss of associability could be revealed in slow acquisition of suppression in Stage 2 in Groups L-L and T-T, for which the target stimulus had been preconditioned in Stage 1, relative to acquisition seen in Groups T-L and L-T, for which the target stimulus was novel. Sessions in Stage 2 were 40 min long, and each contained two 90-sec CS trials beginning after 12 and $35 \mathrm{~min}$ had elapsed. For all groups, Stage 2 continued for three sessions, and the US was a .8-mA 1 -sec scrambled grid shock.

Stage 3: Stage 3 was a search for evidence that Stage 2 had produced weaker conditioning in the groups for which the target CS had been paired with weak shock in Stage 1 than in groups for which the target had been novel. Such evidence could take the form of weaker suppression to the target CSs in the former groups (Groups L-L and T-T) than in their controls (Groups T-L and L-T). Accordingly, Groups L-L and T-L now received light-alone trials, whereas Groups T-T and L-T received tone-alone trials. Following Hall and Pearce (1978), there were two sessions in Stage 3; each was $1 \mathrm{~h}$ long, and each contained four 90-sec CS trials, which began 10.5, 21, 31.5, and $42 \mathrm{~min}$ after the start of the session. Shock USs were not presented.

Response measures: The number of responses occurring $90 \mathrm{sec}$ before and during CS trials were recorded. Suppression during the CS was indexed in terms of the Annau-Kamin (1961) ratio, D/(B + D). Here $B$ denotes the number of responses in the $90-\mathrm{sec}$ period just before each $\mathrm{CS}$, and $\mathrm{D}$ denotes the number during the $\mathrm{CS}$. With this ratio, a score of 0 denotes strong suppression; one of .5 suggests no effect.

\section{Results ${ }^{1}$}

The results of Stage 1 are shown in Figure 1. In general, both the tone and the light CS produced more suppression in this study than in our pilot work; however, once again suppression was stronger for the light (see triangles) than for the tone (see squares). An analysis of variance including all four groups found the effects of groups to be significant $[F(3,28)=9.59, p<.001]$. The two light groups combined suppressed more than did the two tone groups combined $[F(1,28)=26.62, p<.001]$. By Day 2 , every group in the experiment suppressed more on the first trial of the session than on the first trial of Day 1 $[\operatorname{ts}(7)>2.36, \mathrm{ps}<.05]$. This increase in suppression is not an unconditioned novelty effect because such effects

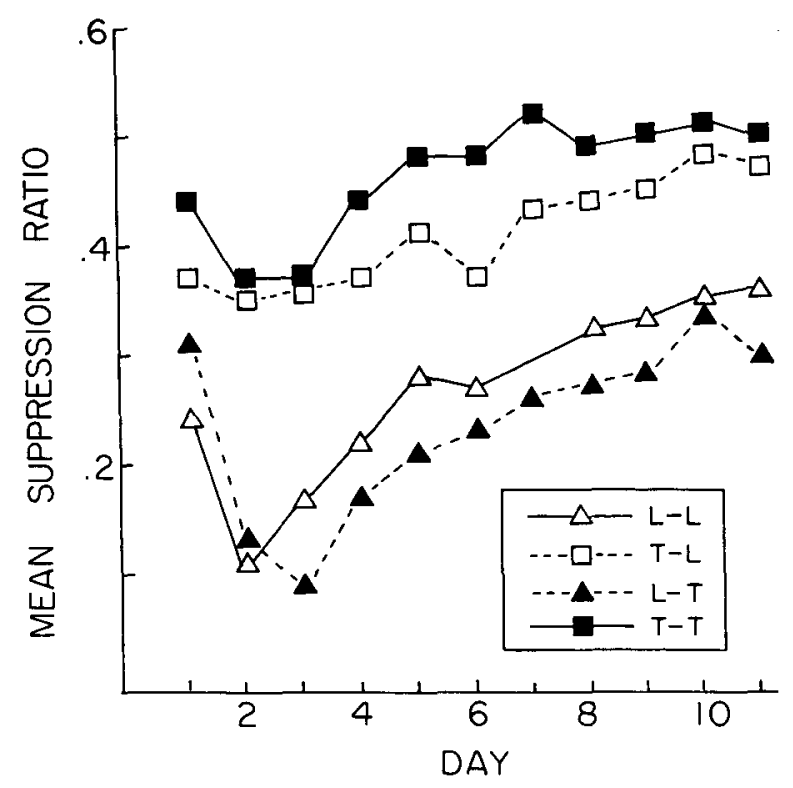

Figure 1. Stage 1: Acquisition of conditioned suppression in Experiment $1 \mathrm{~A}$ with a weak shock US.

habituate with repeated CS presentations; it is therefore suggestive of excitatory Stage 1 conditioning in each group. After reaching asymptote by Day 2 or Day 3, all four groups showed a postasymptotic decline in suppression (cf. Millenson \& Dent, 1971): each group suppressed less on Day 11 than it did on Day $3[\operatorname{ts}(7)>2.96$, ps $<.05]$.

The results from Stages 2 and 3 are presented in Figure 2. The left panel shows the results of the experimental and control groups for which the light was the

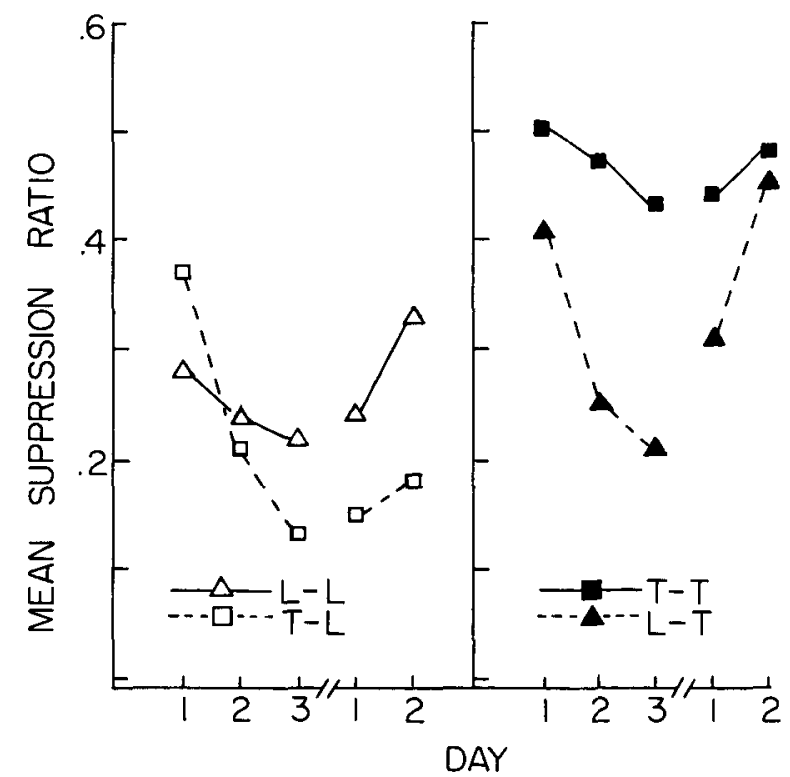

Figure 2. Stages 2 and 3: Acquisition of conditioned suppression in Stage 2 of Experiment $1 \mathrm{~A}$ followed by extinction testing in Stage 3. 
target CS; the right panel shows the results of the experimental and control groups for which the tone was the target CS. In each panel, the data on the left are from Stage 2 and the data on the right are from Stage 3. Figure 2 suggests a greater negative transfer effect in Stage 2 when the tone was the target CS. This impression was supported by a significant interaction $[F(1,28)=4.37, p<.05]$ between the modality of the target CS and group (experimental vs. control). Individual group comparisons performed separately on each day of Stage 2 found that Group T-T yielded significantly weaker suppression than Group L-T on Days 2 and 3 (ps < .05). Similar comparisons between Group L-L and Group T-L yielded no significant differences on any day of Stage 2, although a trend for weaker suppression in the experimental group (Group L-L) relative to its control (Group T-L) appeared on Day 3. However, if the rate of acquisition between the two groups is compared, one finds a slower rate in the experimental group relative to the control group. This observation is also true for Groups T-T and L-T. The impression that experimental groups acquired suppression more slowly than the control groups did was supported by a significant interaction $[F(2,56)=4.20, p<.05]$ between groups (experimental vs. control) and days. And the impression that this effect was similar regardless of the modality of the target CS was supported by a nonsignificant interaction between modality of the target CS, groups, and days $(F<1)$. Thus, although Group L-L did not show significantly less suppression than Group T-L, the smaller increments in suppression over days in Group L-L than in Group T-L suggest that negative transfer did occur even when the light was the target CS.

If this apparent negative transfer effect is real, then it should continue during extinction trials in Stage 3. That is, Group L-L should yield weaker suppression of barpressing compared to Group T-L during extinction. And since Group T-T yielded weaker conditioned suppression than did Group L-T in Stage 2, this pattern too should continue in Stage 3. The figure suggests that, during extinction, both experimental groups did indeed yield weaker suppression than their controls, and this impression was supported by a significant $[F(1,28)=6.76, p<.05]$ effect of groups (experimental vs. control); moreover, this effect did not interact with the modality of the target CS $(\mathrm{F}<1)$.

The top half of Table 1 shows the group mean pre-CS response rates in each stage of Experiment 1 . The pre-CS rates were analyzed using an analysis of variance in which the variables were (1) the modality of the target CS and (2) groups (experimental vs. control). Neither the main effects nor their interaction were significant in any stage.

\section{Discussion}

Experiment 1A demonstrated negative transfer and did so both when the target CS in Stage 2 was a weakly salient auditory stimulus and when it was a more highly salient visual stimulus. There was some evidence (from Stage 2) that the strength of negative transfer was less when the target CS was the more salient visual CS, but there seemed
Table 1

Group Mean Pre-CS Rates (Responses/Min)

\begin{tabular}{lccc}
\hline Group & Stage 1 & Stage 2 & Stage 3 \\
\hline \multicolumn{4}{c}{ Experiment 1 } \\
L-L & 25 & 30 & \\
T-L & 15 & 19 & 29 \\
T-T & 25 & 28 & 17 \\
L-T & 18 & 27 & 29 \\
& Experiment 2 & 26 \\
L.4-L.8 & 25 & 27 & \\
N.4-L.8 & 20 & 25 & 31 \\
L1.0-L1.3 & 15 & 24 & 25 \\
N1.0-L1.3 & 15 & 24 & 21 \\
\hline
\end{tabular}

to be no difference in the strength of transfer as assessed in Stage 3. (See also the strong negative transfer with the visual target CS in Stage 2 of Experiment 2A to follow.) The conclusion seems to be that the negative transfer effect is both general and robust; more importantly, it occurs even when, in Stage 1, conditioned suppression to the target CS is moderately strong. Of course, that moderately strong suppression did show a postasymptotic decline in Stage 1 as CS-US pairings continued beyond Day 3. For this reason we sought in Experiment $2 \mathrm{~A}$ to produce even stronger suppression in Stage 1 to the target CS and perhaps to eliminate the postasymptotic decline. We wanted to find out whether negative transfer would still occur.

\section{Experiment 2A}

Experiment $2 \mathrm{~A}$ sought to determine whether negative transfer would still occur even if experimental groups showed moderate to strong levels of suppression in Stage 1. To this end, one experimental group, Group L.4-L.8, was conditioned with the flashing light CS in Stages 1 and 2, because this stimulus yielded moderate levels of suppression in Experiment $1 \mathrm{~A}$ even under low levels of shock. The control group, Group N.4-L.8, received an intermittent noise CS during Stage 1 and the light CS in Stage 2. Both groups were trained using the 4 -mA shock in Stage 1 and the .8-mA shock in Stage 2. These groups were similar, then, to the L-L and T-L groups of Experiment 1, in which Group L-L showed negative transfer despite showing moderately strong suppression to the target CS in Stage 1. A second pair of groups was included to determine whether negative transfer could be obtained if the CS produced strong suppression in Stage 1. These groups, Groups L1.0-L1.3 and N1.0-L1.3, received a shock of $1.0 \mathrm{~mA}$ for $1 \mathrm{sec}$ in Stage 1 and a shock of $1.3 \mathrm{~mA}$ for $1.5 \mathrm{sec}$ in Stage 2. For the experimental group, Group L1.0-L1.3, the combination of light CS and 1-mA shock US in Stage 1 was expected to produce strong suppression to the CS in that stage. The question was: Would negative transfer still occur under this condition?

\section{Method}

Subjects and Apparatus. Thirty-two male albino rats, similar to those of Experiment $1 \mathrm{~A}$ served as experimental subjects and were maintained as before. The apparatus was unchanged. 
Procedure. Except where noted, the procedures were the same as in Experiment 1A. Following preliminary training, each rat was assigned to one of four groups: N.4-L.8, L.4-L.8, N1.0-L1.3, and L1.0-L1.3 (N $=8$ /group). The groups were matched in terms of body weight and rate of responding during preliminary VI training. Conditioning chambers were balanced across groups. In each group designation, the letter before the dash denotes the stimulus that was paired with the weak shock in Stage 1 and the number indicates the intensity of the shock. The letter and number after the dash denote, respectively, the type of CS and the intensity of shock in Stage 2. Thus, Groups N.4-L.8 and L.4-L.8 received .4-mA .5-sec shocks in Stage 1 and .8-mA 1-sec shocks in Stage 2. Groups N1.0-L1.3 and L1.0-L1.3 received $1.0-\mathrm{mA} 1.0$-sec shocks in Stage 1 and 1.3-mA 1.5-sec shocks in Stage 2. The letter $\mathbf{N}$ denotes an intermittent (1-sec-on, .11-sec-off) white noise CS of 80-dB intensity re $20 \mu \mathrm{N} / \mathrm{m}^{2}$; the letter L denotes the flashing light CS of Experiment $1 A$. Stage 1 had 11 daily sessions with six trials per day. Stage 2 had 3 daily sessions with two trials per day. Stage 3 had 2 daily sessions of four light-alone trials.

\section{Results}

The results of Stage 1 are shown in Figure 3. A CS $\times$ shock level $\times$ days $\times$ trials analysis of variance confirmed the impression conveyed by Figure 3 that suppression increased as a function of shock intensity $[\mathrm{F}(1,28)=74.35$, $\mathrm{p}<.001]$ and that the light CS produced more suppression than did noise $[F(1,28)=8.20, p<.01]$. By Day 2 , every group in the experiment suppressed more on the first trial of the session than on the first trial of Day 1 $[\operatorname{ts}(7)<2.36$, ps $<.05]$. This increase in suppression with training is suggestive of Stage 1 conditioning in each group. Both groups receiving the lower intensity US (.4 mA) reached asymptote on Day 2 , and both showed a postasymptotic decline in suppression [Day 2 vs. Day 11: $\operatorname{ts}(7)>2.36$, ps < .05]. At the higher intensity, both groups reached asymptote between Days 3 and 4 and showed only a nonsignificant decline in suppression thereafter. Thus, it appears that the postasymptotic decline was greater with weak USs.

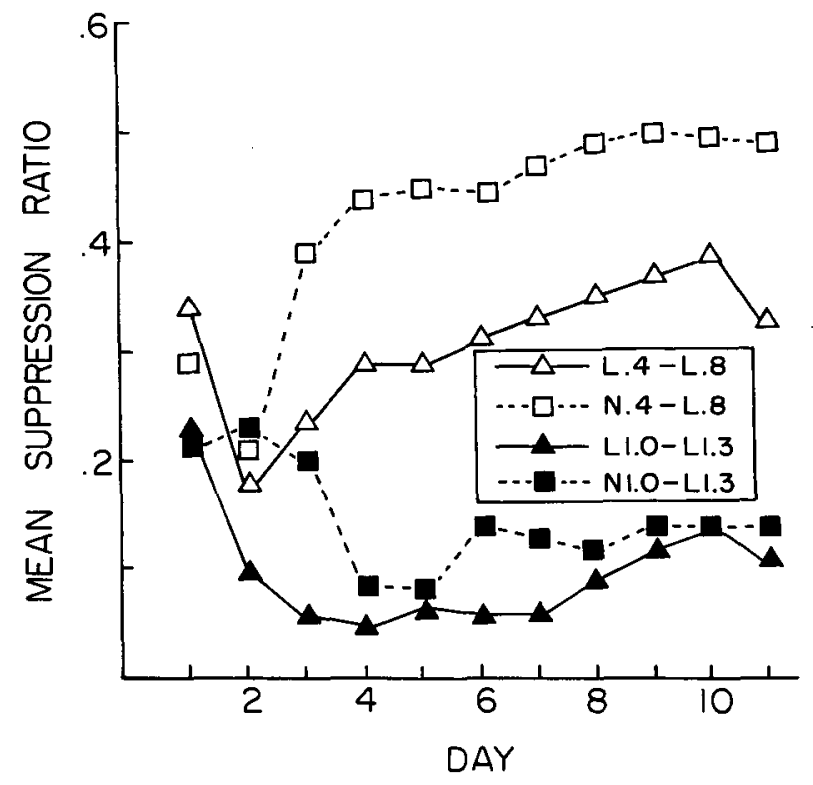

Figure 3. Stage 1: Acquisition of conditioned suppression in Experiment $2 \mathrm{~A}$.

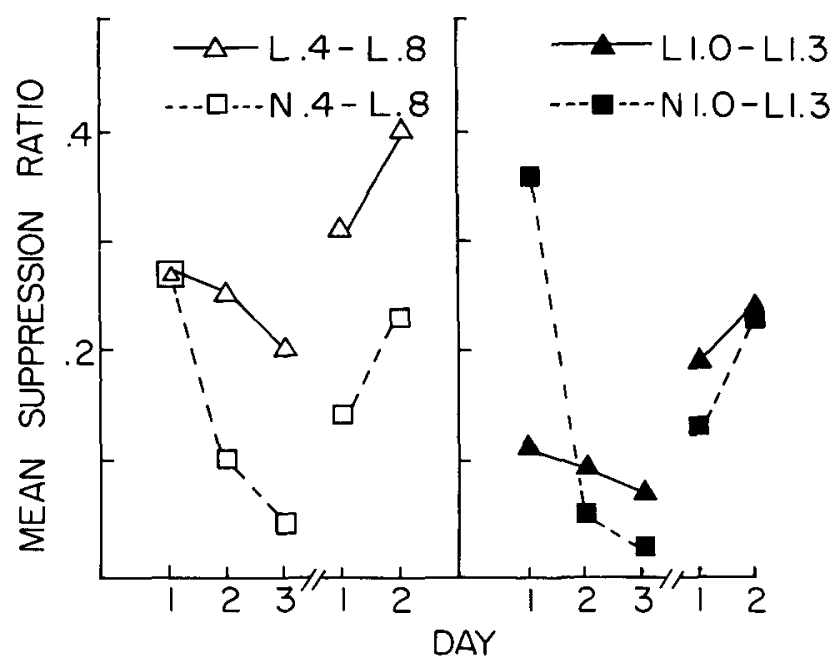

Figure 4. Stages 2 and 3: Acquisition of conditioned suppression in Stage 2 of Experiment $2 A$ with a light CS and strong shock US followed by extinction testing in Stage 3. For groups in the left panel, the US was a 8-mA 1-sec shock; for groups in the right panel, the US was a 1.3-mA 1.5-sec shock.

Figure 4 presents the results from Stages 2 and 3. The left panel shows the data for the groups that received the .8-mA US in Stage 2; the right panel shows the data for the groups given the 1.3-mA US. In each panel, the data from Stage 2 are on the left and the data from Stage 3 are on the right. The figure suggests that in Stage 2, for the .8-mA groups, the retardation effect reported by Hall and Pearce (1979) was again observed. This impression was confirmed by a groups $\times$ days $\times$ trials analysis of variance that found the groups $\times$ days interaction $[F(2,28)$ $=7.89, \mathrm{p}<.05$ ] to be statistically significant. Individual $t$ tests showed that Group L.4-L.8 suppressed significantly less than Group N.4-L.8 on Days 2 and 3 (ps $<.05]$.

The right panel of Figure 4 suggests that, under the higher shock intensities, the experimental group showed positive transfer rather than negative transfer. A groups $x$ days $x$ trials analysis of variance found a significant groups $\times$ days interaction $[\mathrm{F}(2,28)=64.71, \mathrm{p}<.001]$. The interaction reflected significantly more suppression in Group L1.0-L1.3 than in Group N1.0-L1.3 on Day 1 $(\mathrm{p}<.01)$ and no significant difference between these two groups on Days 2 and $3(p>.05)$. Any real difference between the two groups on Days 2 and 3, however, may have been masked by a ceiling of suppression. The purpose of Stage 3 was to search for evidence of weaker suppression in the experimental groups relative to the controls under conditions less likely to be affected by ceiling effects. Since a retardation effect was observed in Stage 2 for the .4-mA condition, this effect should also be observed in Stage 3. The left-hand panel of Figure 4 clearly suggests weaker suppression in Stage 3 in Group L.4-L.8 than in Group N.4-L.8. This observation is supported by a groups $\times$ days $\times$ trials analysis of variance, which found a significant main effect of groups $[F(1,14)=7.52$, 
$p<.05]$. The right panel in Figure 4 also suggests weaker suppression in Group L1.0-L1.3 than in Group N1.0-L1.3, at least on the 1st day of Stage 3. A groups $\times$ days $x$ trials analysis of variance, however, found a nonsignificant effect of groups $(F<1)$. An individual $t$ test on Day 1 also failed to find a significant difference between groups $(p>.10)$.

The bottom half of Table 1 shows the group mean pre$\mathrm{CS}$ rates in each stage of Experiment 2A. The pre-CS rates from each stage were analyzed using an analysis of variance in which the factors were (1) modality of the target $\mathrm{CS}$ and (2) shock intensity. Only the effect of shock intensity was significant $[F(1,28)=4.70, p<.05]$, and that only in Stage 1. Rats receiving the 1.0-mA shock in that stage had lower pre-CS rates than those receiving the 4-mA shock.

\section{Discussion}

In this experiment negative transfer was observed again, both in Stage 2 and Stage 3, when moderate levels of suppression to the CS were seen in Stage 1. However, when suppression in Stage 1 was strong, positive transfer rather than negative transfer was produced.

\section{General Discussion of Part A}

These experiments, using conditioned suppression, have successfully replicated the negative transfer effect originally described by Hall and Pearce (1979). In the present work, the effect occurred under a variety of CS and US conditions: it occurred both when the target CS in Stage 2 was a nonsalient auditory CS and when it was a highly salient visual CS. It occurred both when the CS in Stage 1 produced very weak levels of suppression and when it produced moderately strong levels followed by a postasymptotic decline in suppression. It did not occur, however, when the suppression in Stage 1 was very strong and was not followed by a postasymptotic decline; under that condition, positive transfer occurred instead.

It is important to note that our findng of positive transfer in Experiment 2A is not incompatible with the Pearce and Hall model. We have already shown in the introduction that the model can predict positive as well as negative transfer; and we have shown that positive transfer should tend to occur when there is little difference in the asymptotes of Stage 1 and Stage 2 conditioning. This seems to be what Experiment $2 \mathrm{~A}$ has demonstrated.

However, it is also important to note that loss of CS associability is not the only mechanism through which negative transfer might be produced. Negative transfer could also result if the US, rather than the CS, lost its effectiveness. According to at least one theory (Wagner, 1976, 1978), this would be more likely to happen in the experimental group than in the control because only for the experimental group would the US representation (or an approximation to it) be primed in short-term memory by the target CS at the start of Stage 2. According to the theory, such priming should reduce the US effectiveness.
We wish to note also that both the Pearce and Hall model and Wagner's theory agree that some event (be it CS or US) loses effectiveness because of an associative process. In the Pearce and Hall model, the CS loses associability because it reliably predicts its consequences or, more formally, because $V_{A}$ approaches $\lambda$. In Wagner's theory, the US loses effectiveness because its representation in shortterm memory is primed by a CS associated with the US. An alternative to these explanations is that the negative transfer that occurs when a CS is paired with a weak US is not a consequence of an associative process involving the US, but is instead merely a latent inhibition process attributable to CS preexposure. That is, it is possible that the Hall and Pearce negative transfer effect occurs not because the CS predicts the US but, rather, despite the fact that it predicts the US (cf. Lubow et al., 1981). We are suggesting, in short, that a balance exists between processes that produce negative transfer (latent inhibition due to CS preexposure) and those that produce positive transfer (conditioning due to CS-US pairings) (cf. Rudy \& Cheatle, 1978). When conditioning is very weak, as in Experiment $1 \mathrm{~A}$, the latent inhibition that occurs because of repeated CS presentations is sufficient to produce negative transfer. But when conditioning is very strong, as in Experiment $2 \mathrm{~A}$, the gains in associative strength outweigh the gains in latent inhibition, resulting in positive rather than negative transfer. These two cases lie at extreme ends of a continuum. Intermediate is the case in which conditioned performance is initially moderate in strength but then weakens postasymptotically. Moderate conditioning may or may not be sufficient to outweigh latent inhibition; the postasymptotic decline in performance, however, increases the likelihood that latent inhibition wins out.

In the present experiments, as in the Hall and Pearce experiments after which ours were patterned, experimental rats received a total of 5,940 sec of exposure to the target CS in Stage 1 prior to target conditioning in Stage 2. Control rats, in contrast, received no exposure to the target CS prior to target conditioning. According to the Pearce and Hall model, the crucial dimension along which the two groups differed just prior to Stage 2 was the extent to which the target CS had previously predicted its consequences. This extent was considerable for the experimental group and zero for the control. But, clearly, there was in these experiments an enormous confound between this factor (CS predicts consequence) and the factor of familiarity with the target CS (familiarity being defined in terms of the total amount of time the target CS was preexposed). Which of these two factors was responsible for negative transfer? We shall return to this question in the discussion of Part B to follow.

\section{PART B: NICTITATING MEMBRANE RESPONSE (NMR)}

Experiments with the rabbit NMR also used the twostage transfer-of-training design described in Part A. In Stage 1, one group of subjects received a series of CS-US 
pairings designed to reduce the associability of the target CS. A second group received a comparable series of CSUS pairings, but the CS was not the target CS. In Stage 2, both groups recieved pairings of the target $\mathrm{CS}$ with a strong US.

As noted in the introduction, Pearce and Hall's model predicts that a group that experiences the target CS in Stage 1 should be comparatively slower to condition in Stage 2, and should attain a lower level of conditioned responding, than a group that experiences the other CS in Stage 1. To enhance the negative transfer effect predicted by Pearce and Hall, most of the experiments described below kept the US intensity in Stage 1 at the absolute minimum necessary to elicit a detectable unconditioned response (UR) and used a much stronger US intensity in Stage 2.

In Stage 1, US intensity (electrostimulation of the periocular region of the right eye-eyeshock, for short) was typically just above the threshold current level necessary to elicit a UR. This UR was only a small fraction of the amplitude of a full-scale UR, as low as $5 \%$ in individual cases. In most of the experiments, Stage 1 current levels were continuously monitored and adjusted for each animal to ensure elicitation of a UR throughout this stage. Without this precaution, Stage 1 would have included within it CS presentations with no detectable consequence. These would be tantamount to CS-alone trials. A significant number of such occurrences would produce extinction of whatever conditioning might have occurred up to that point. According to the Pearce-Hall model, extinction procedures tend to reinstate lost associability of the CS. This consequence would, of course, work against negative transfer as envisioned in this theory. Alternatively, CSalone occurrence would contribute to negative transfer in an unintended way by engaging mechanisms responsible for latent inhibition. In Stage 2, the current was increased to a level that would evoke a full-scale UR. This level was, on the average, five times that of Stage 1, but was constant for all animals within a given experiment. It could be argued that adjustments of Stage 1 current to mitigate these problems have the unintended effect of reinstating associability presumed to be lost through CS-US pairing. This concern may imply that tests of the Pearce-Hall model cannot be implemented in circumstances in which unconditioned responses to the weaker US are demanded.

With these and other procedures, the following series of experiments failed to detect the negative transfer predicted by Pearce and Hall's model. Instead, the evidence supported the conclusion that Stage 1 pairings promoted acquisition, and this learning carried over to Stage 2 to yield a positive transfer effect. Several strategies were employed to reduce conditioned performance in Stage 1 prior to the shift to Stage 2. When these strategies succeeded, either positive transfer (as before) or no transfer was the result. By positive transfer, we mean that groups receiving Stage 1 training with the target CS gave more CRs in Stage 2 than did controls. Their rate of conditioned responding in Stage 2 began at a higher level and converged monotonically toward the ceiling of $100 \%$ CRs. At no point in
Stage 2 did the acquisiton curve for control groups exceed that of the corresponding experimental group.

\section{Experiment 1B}

Our initial attempt to demonstrate negative transfer through loss of associability in the two-stage design employed experimental parameters similar to those used in previous demonstrations of latent inhibition in this laboratory (e.g., Solomon \& Moore, 1975), including the use of 450 Stage 1 trials, matching the number of CS exposures that has routinely yielded a robust latent inhibition effect in this laboratory.

\section{Method}

Subjects and Apparatus. Eight naive albino rabbits (Oryctolagus cuniculus), obtained from a licensed supplier, served as subjects. They were housed individually with ad-lib access to food and water. The colony room was under constant illumination . Apparatus and ancillary procedures have been described in previous published reports (e.g., Berthier $\&$ Moore, 1980). The light CS consisted of the onset of two 4.5-V incandescent lights (see Berthier \& Moore, 1980), and the tone CS was a $1200-\mathrm{Hz}$ sine wave of $90-\mathrm{dB}$ re: $20 \mu \mathrm{N} / \mathrm{m}^{2}$. The US was a $60-\mathrm{Hz}$ ac electrical pulse of 50-msec duration delivered via wound-clip electrodes affixed to the skin of the periocular region of the right eye. Responses were recorded via a rotary potentiometer and displayed on an oscillographic recorder (see Gormezano, 1966). Conditioned responses were defined as deflection of the recording pen within the CS-US interval of at least $1 \mathrm{~mm}$ from pretrial baseline. This corresponds to a movement of the nictitating membrane of $.5 \mathrm{~mm}$, approximately $5 \%$ of its full extension. Subjects were run four at a time in ventilated darkened chambers.

Procedure. For all subjects, training consisted of 100 trials per daily session. The CS-US interval was constant $(.5 \mathrm{sec})$ with the CS and US terminating together. The intertrial interval was also constant at $30 \mathrm{sec}$. Stage 1 consisted of 4.5 sessions ( 450 trials), and the transition to Stage 2 occurred without interruption midway through the fifth session. An additional 50 Stage 2 trials were given in the sixth session, by which time all eight subjects had attained a response ceiling of over $90 \% \mathrm{CRs}$. Because the tone was the preconditioned CS for Group T-T, all subjects received this CS during Stage 2. It was paired with a 1.5-mA eyeshock, which was the Stage 2 US.

Four subjects (Group T-T) received the tone paired with weak eyeshock in Stage 1. The other four subjects (Group L-T) received the light CS paired with weak shock. Stage 1 shock level was continuously adjusted during Stage 1 so as to maintain detectable URs from all subjects. The average current levels at the end of Stage 1 were .18 and $.24 \mathrm{~mA}$ for Groups T-T and L-T, respectively.

\section{Results and Discussion}

Negative transfer would have manifested itself as more conditioned responding in Stage 2 for Group L-T than for Group T-T. This, however, did not occur: Group L-T gave only $73 \%$ CRs in Stage 2, whereas Group T-T gave 91\% (Mann-Whitney $\mathrm{U}=3$; one-tailed $\mathrm{p}=.10$ ). The advantage of Group T-T over Group L-T began in Stage 1, in which the former gave an overall average of $38 \%$ CRs and Group L-T gave one of $20 \%(\mathrm{U}=3, \mathrm{p}=.10)$.

This difference between the two groups in Stage 1 was not unexpected because the tone is the more salient of the two CSs, based on prior research. What was surprising, however, was the relatively high frequency of conditioned responses supported by shock levels just sufficient to elicit URs. This high level of responding in Group T-T is particularly unfortunate because it introduced compli- 
cations in how Stage 2 response levels bear on the question of negative transfer: By one definition, negative transfer occurred because Group L-T was able to obtain as high a terminal level of responding ( $79 \% \mathrm{CRs)}$ as Group T-T within only 100 trials, all in Stage 2, whereas Group T-T required over 500 trials to attain this level, counting the $\mathbf{4 5 0}$ trials of Stage 1 . This is not an altogether satisfactory definition of negative transfer, as one would like to have had the two groups enter Stage 2 with low and roughly comparable starting points so as to allow transfer effects to emerge within the full range of the performance measure, percentage of CRs.

Experiment $1 \mathrm{~B}$ was repeated with eight naive animals and with one change: ${ }^{2}$ The CS-US interval was increased for both stages of training from 500 to $1,500 \mathrm{msec}$ in an attempt to mitigate high levels of Stage 1 conditioned responding. This tactic was not altogether successful, since Group T-T gave 55\% CRs over the 50 trials just prior to Stage 2 and showed positive transfer relative to Group L$\mathrm{T}$ in that stage.

\section{Experiment 2B}

Experiment 2B used a trace conditioning procedure in an attempt to further reduce Stage 1 performance by Group T-T. Trace conditioning should also have imposed greater demands than delay conditioning on informationprocessing mechanisms responsible for reducing associability. Our intuition, based on failure to observe negative transfer in Experiment 1B, was that a temporal separation between the CS and US might prevent premature interruption of processing of the CS by the occurrence of the US. Such processing is presumably necessary for the loss of associability through acquisition.

\section{Method}

Eight naive rabbits served as subjects; four were assigned to Group T-T and four to Group L-T. The CSs in both stages were on for $250 \mathrm{msec}$, and the trace interval was $500 \mathrm{msec}$, yielding a CS-US interval (onsetonset) of $750 \mathrm{msec}$. Stage 1 terminal shock levels averaged $.46 \mathrm{~mA}$ for Group T-T and $.43 \mathrm{~mA}$ for Group L-T. All other procedural details were the same as in Experiment 1B.

\section{Results and Discussion}

Group T-T gave $94 \%$ CRs over the course of 150 Stage 2 trials, with all four subjects over $90 \%$. By contrast, Group L-T subjects gave only $21 \%$ CRs to the tone over these trials $(U=0, p=.01)$ and never exceeded $50 \%$ CRs as a group over the course of an additional 250 trials.

To check on the possibility that Group L-T was inadvertently composed of subjects incapable of high-level conditioned responding, we gave a series of 200 acquisition trials in the trace paradigm to Group L-T using the light CS they had experienced in Stage 1. With the $1.5-\mathrm{mA}$ shock as the US, all subjects in Group L-T gave over $90 \%$ CRs on the second 100-trial session. Thus, these subjects were perfectly capable of high levels of conditioned responding.
This experiment clearly failed to demonstrate negative transfer. However, the trace procedure was not altogether successful in eliminating responding to the tone in Stage 1 for Group T-T. Although Group T-T averaged only $17 \%$ CRs over the 450 Stage 1 trials, these subjects had $58 \%$ CRs as a group over the last 50 trials of Stage 1 just prior to the transition to Stage 2 .

The trace conditioning procedure of Experiment $2 \mathrm{~B}$ was used with eight naive rabbits in a follow-up study in which only 300 Stage 1 trials were presented instead of 450 as in Experiments $1 \mathrm{~B}$ and $2 \mathrm{~B}$. With fewer Stage 1 trials, Group T-T entered Stage 2 after having given only $17 \%$ CRs on the last session of Stage 1. Once again, however, in Stage 2, significant $(U=1, p=.03$ ) positive transfer occurred in Group T-T relative to Group L-T.

\section{Experiment 3B}

Like Experiment 2B, Experiment 3B also used a trace conditioning procedure, but the light, rather than the tone, served as the target CS.

\section{Method}

Eight naive rabbits served as subjects, with four assigned to each of two groups as in previous experiments. Procedures were exactly the same as in Experiment 2B except that Stage 2 consisted of $300 \mathrm{CS}$-US trials to the light CS (100/day) and the light served as the target CS. Stage 1 terminal shock levels averaged $.14 \mathrm{~mA}$ for Group T-L and .51 $\mathrm{mA}$ for Group L-L.

\section{Results and Discussion}

Despite the unusually high levels of current needed for detectable URs in Stage 1, Group L-L did not give any CRs during the 300 trials of this stage. Group T-L, by contrast, gave $11 \%$ CRs overall, with $20 \%$ CRs on the last 100 trials of Stage 1.

As in the previous experiment, there was no evidence of negative transfer with respect to the light CS. During Stage 2, Group L-L gave $64 \%$ CRs, but Group T-L gave only $28 \%(\mathrm{U}=1, \mathrm{p}=.03)$.

\section{Experiment 4B}

In a further effort to detect evidence of negative transfer, Experiment 4B introduced several procedural departures from the previous experiments. Foremost, the number of Stage 1 trials was reduced from 300 to 150 , the intertrial interval was reduced from 30 to $15 \mathrm{sec}$, and Stage 1 shock levels were determined prior to CS-US pairings and held constant for individual subjects. Tone served as the target CS in a forward delay procedure.

\section{Method}

Eight naive rabbits were subjects, with four assigned to each of two groups. All subjects received a series of 40 US-alone presentations, during which a shock level was selected that would consistently elicit a detectable UR. These shock levels, averaging .35 and $.47 \mathrm{~mA}$ for Groups T-T and L-T, respectively, remained constant for the $150 \mathrm{CS}$-US pairings of Stage 1, which was initiated on the following day. As mentioned above, 
the intertrial interval was $15 \mathrm{sec}$, and a forward delay paradigm was employed, as in Experiment 1B, with a CS-US interval of $.5 \mathrm{sec}$. Stage 2 shock level was $1.5 \mathrm{~mA}$. Stage 2 began midway through the second session of CS-US pairings; an additional two daily sessions of Stage 2 were run, making a total of 250 trials.

\section{Results and Discussion}

As in Experiment 1B, Group T-T attained a high level of conditioned responding prior to Stage 2, averaging $57 \%$ CRs over the 50 trials preceding the switch. Furthermore, although Group T-T gave $91 \%$ CRs in Stage 2, Group L$\mathrm{T}$ gave only $62 \%(\mathrm{U}=2, \mathrm{p}=.06)$. Thus, there was no evidence of negative transfer.

A repetition of Experiment 4B was performed with a tone CS less intense $(80 \mathrm{~dB}$ instead of $90 \mathrm{~dB})$ than that used in previously described experiments. Reducing the intensity of the target CS resulted in the complete elimination of Stage 1 CRs. Nevertheless, there was no evidence of negative transfer, inasmuch as Group L-T gave $72 \%$ CRs in Stage 2 and Group T-T gave $78 \%$.

\section{Experiments 5B and 6B}

Using a variety of parametric manipulations, the previous experiments failed to provide evidence of negative transfer. The amount of Stage 1 training varied from 150 to 450 trials. At this point, it appeared likely that negative transfer might occur with fewer Stage 1 trials. Pearce and Hall's (1980) equations suggest that losses of associability should be most pronounced with the first few Stage 1 pairings of the CS and US. We therefore reasoned that reducing the number of Stage 1 trials might reveal evidence of negative transfer, which had been obscured by too much conditioned performance in the previous experiments. Accordingly, Experiments $5 \mathrm{~B}$ and $6 \mathrm{~B}$ used only 50 Stage 1 trials.

\section{Method}

Eight naive rabbits were assigned to each experiment, half designated as Group T-T and half as Group L-T. Both experiments used precisely the same procedures as Experiment 4B, except that only 50 Stage 1 trials were given, the switch to Stage 2 occurring midway through the first session. Subjects in Experiment 5B went on to receive a total of $\mathbf{3 0 0}$ Stage 2 trials; subjects in Experiment 6B received 150 Stage 2 trials. Other than this, Experiments 5B and 6B differed only in the intensity of the tone CS, which was $80 \mathrm{~dB}$ for the former and $90 \mathrm{~dB}$ for the latter.

Stage 1 shock levels in Experiment 5B averaged .21 mA for Group T-T and $.19 \mathrm{~mA}$ for Group L-T. In Experiment 6B, these corresponding shock levels were $.07 \mathrm{~mA}$ for Group T-T and .11 $\mathrm{mA}$ for Group L-T.

\section{Results and Discussion}

With only 50 trials, no CRs occurred in Stage 1 in either experiment. In Experiment 5B, Group T-T had an overall average of $66 \%$ CRs in Stage 2 and Group L-T one of $59 \%$ CRs $(\mathrm{U}=6, \mathrm{p}=.34)$. In Experiment $6 \mathrm{~B}$. these corresponding figures were $79 \%$ for Group T-T and $71 \%$ for Group L-T $(U=4, p=.17)$. Thus, no evidence of negative transfer emerged from either experiment.

A repetition of Experiment $6 \mathrm{~B}$ with only 25 Stage 1 trials also failed to reveal negative transfer, showing marginally significant $(p=.06)$ positive transfer instead.

\section{Experiments 7B and 8B}

Apart from Experiment 4B, in which Stage 1 shock levels were set prior to Stage 1, all of the preceding experiments involved continuous monitoring and adjusting of Stage 1 shock levels so as to maintain, as nearly as possible, detectable URs. This procedure can be justified because fixed shock at a level well above the threshold promotes too much Stage 1 responding, thereby obscuring any possible negative transfer. Lower fixed shock levels would typically elicit URs for a few Stage 1 CS-US pairings, but the UR would quickly habituate to the point where detectable URs would not occur. This being the case, the CS-US pairings of Stage 1 would be tantamount to a series of CS-alone trials. Any resulting negative transfer could then be as easily attributed to latent inhibition as to loss of associability through acquisition. Nevertheless, the continual fine tuning of Stage 1 shock levels might be cited as contributing to the failure to observe negative transfer.

Another factor that could have affected the direction of transfer was the actual shock intensity used in Stage 1. Subjects trained in Stage 1 to the tone usually required a lower current level (mean $=.22 \mathrm{~mA} ; \mathrm{SD}=.18$ ) to maintain URs than those trained to the light (mean $=.34 \mathrm{~mA}$; SD $=.20$ ). The explanation for this difference may be found in reports of reflex facilitation in the presence of acoustic stimuli (e.g., Ison \& Leonard, 1971). It is possible that the Stage 2 US was perceived by subjects in Group L-T as less intense than would have been the case if they had not become accustomed to the comparatively higher level of shock in Stage 1. This adaptation could have rendered Stage 2 shock less effective for subjects in Group L-T than for those in Group T-T, thereby contributing to slower acquisition in Stage 2.

Experiments 7B and 8B fixed the Stage 1 shock level for all subjects in order to eliminate the contribution of the two factors discussed in the preceding paragraphs. In addition, training protocols were modified such that only $\mathbf{5 0}$ trials were given per session to check on the possibility that trials per session contributes to the direction of transfer in the two-stage design. Both experiments used only 25 Stage 1 trials in order to avoid the high levels of conditioned responding that would occur using the level of shock selected for Stage 1-a level high enough to ensure fullscale URs.

\section{Method}

Eight naive rabbits were assigned to each experiment, four to Group T-T and four to Group L-T. The procedures were the same as in Experiment $6 \mathrm{~B}$, except for the following: Stage 1 shock level was $.5 \mathrm{~mA}$ for all subjects (1.5 $\mathrm{mA}$ in Stage 2); 50 trials, instead of 100 , were given per session, and the intertrial interval was $30 \mathrm{sec}$. In Experiment 7B, 175 trials were given in Stage 2. Because of rapid learning, only 75 Stage 2 trials were givon in Experiment 8B.

\section{Results and Discussion}

In Experiment 7B, Group L-T gave 61\% CRs in Stage 2, and Group T-T gave $49 \%(U=6, p=.34)$. There were no CRs recorded for either group in Stage 1. Although 
statistically nonsignificant, the small negative transfer observed in this experiment dictated Experiment 8B. In the latter, Group T-T gave $61 \%$ CRs over the 75 Stage 2 trials and Group L-T gave $49 \%$. Thus, there was no negative transfer in this experiment $(\mathrm{U}=2, \mathrm{p}=.20)$.

Subjects in Experiment 8B went on to receive 110 conditioning trials with light as the target CS paired with the 1.5-mA eyeshock. Here, Group L-T gave more CRs to the target CS $(87 \%)$ than did Group T-T (70\%), suggesting positive transfer from Stage 1 rather than negative transfer $(\mathrm{U}=1, \mathrm{p}=.03)$.

\section{General Discussion of Part B}

The experiments using the rabbit NMR procedure failed to demonstrate negative transfer; instead, they usually demonstrated positive transfer. Once again, the demonstration of positive transfer per se poses no problem for the Pearce and Hall model. What is a problem is the fact that the US intensities used in Stage 1 of the preceding experiments were as weak as possible, consistent with our intent to produce measurable URs in Stage 1. We can thus assume that the Stage $2 \lambda$ was much larger than the Stage 1 $\lambda$. Since this is a condition held to be favorable for demonstrating negative transfer according to the Pearce and Hall model, our results with the NMR procedure were not anticipated by the model.

According to the Pearce and Hall model, a target CS loses associability in Stage 1 because it reliably predicts its consequences (mathematically because $V_{A}$ approaches $\lambda)$. Indeed, much of the appeal of the Pearce and Hall model lies in its use of this single principle, not only to explain negative transfer, but also to explain Kamin blocking, negatively accelerated learning curves, and conditioned inhibition. It is worth noting, however, that all of these latter phenomena have been demonstrated in this laboratory using NMR procedures similar to those used here (Marchant, Mis, \& Moore, 1972; Marchant \& Moore, 1973). Why should these latter phenomena, but not negative transfer, be so easily demonstrated with the NMR procedure? One possibility that should be considered seriously in our view is that negative transfer is produced by a totally different mechanism; that is, it has little to do with $V_{A}$ approaching $\lambda$.

If negative transfer is not due to $V_{A}$ approaching $\lambda$, then what is it due to and why did it occur in our conditioned suppression experiments and not in our NMR experiments? One possibility is that negative transfer is due simply to CS familiarity. By CS familiarity, we refer to the total duration of target CS exposure that the organism receives prior to target conditioning in Stage 2 of the transfer paradigm. It is CS exposure and not CS predicting consequence, we suggest, that produces negative transfer. Note that in the Hall and Pearce transfer design, these two factors are confounded: Although it is true that the CS comes to predict its consequences in Stage 1 only for the experimental group and not for the control group, it is also true that the total exposure to that target CS is greater for the experimental group than it is for the control group. Indeed, in the conditioned suppression experiments of Hall and Pearce (1979) and in our present suppression experiments, the experimental groups received 5,940 sec (66 trials $\times 90 \mathrm{sec} /$ trial) of exposure to the target CS prior to Stage 2, whereas controls received none. This is a serious confound between simple CS exposure and CS predicting consequence. In contrast, in the NMR procedure used in the present report, this confound was not as serious. To be sure, for the experimental groups there were many pairings of the target CS with the US so that the CS well predicted its consequence. However, CS durations were at most only $1.55 \mathrm{sec}$ in the NMR experiments. The greatest amount of target CS exposure in Stage 1 for experimental groups in the NMR experiments was $698 \mathrm{sec}$ (450 trials $\times 1.55 \mathrm{sec} / \mathrm{trial})$. Control groups, of course, received no target $\mathrm{CS}$ exposure in Stage 1. Thus experimental groups in the suppression experiments received over eight times as much target preexposure as did the experimental groups in the NMR experiments. Perhaps this difference in total CS preexposure contributed to negative transfer in the suppression experiments and to the lack of it in the NMR experiments.

We suggest, then, that negative transfer might be a latent inhibition effect due to CS familiarity and not due to CS predicting consequence. Thus, negative transfer occurs despite the fact that the CS predicts a consequence, not because it predicts a consequence (for a compatible theoretical view, see Lubow et al., 1981). We suggest further that there is a balance between latent inhibition processes due to CS preexposure and conditioning processes due to CS-US pairings. If there is too much conditioning in Stage 1, as there was in the high shock groups of Experiment 2A, then positive, not negative, transfer results. In contrast to the Pearce and Hall model, we propose that negative transfer, Kamin blocking, negatively accelerated learning curves, and conditioned inhibition are not all produced by the same factor. We agree with Pearce and Hall and with other theorists (e.g., Rescorla \& Wagner, 1972) that Kamin blocking, negatively accelerated learning, and conditioned inhibition depend in some way on $\mathrm{V}_{\mathrm{A}}$ approaching $\lambda$; but, unlike Pearce and Hall, we suggest that negative transfer depends only on total CS preexposure. This is why all the other phenomena were readily demonstrated in the NMR situation in this laboratory (Marchant et al., 1972; Marchant \& Moore, 1973), but negative transfer was not.

To our knowledge, there is only one line of evidence suggesting that a CS loses associability because it reliably predicts its consequences. This is the finding (Hall \& Pearce, 1982) that presenting the CS alone a few times between Stages 1 and 2 attenuates the negative transfer effect. According to the model, this occurs because the CS-alone presentations cause some extinction of the associative strength acquired in Stage 1. Extinction produces a larger discrepancy between $\lambda$ and $V$ on entering reinforced Stage 2 training than would have been the case had this extinction not taken place, and this increased dis- 
crepancy restores CS effectiveness. However, this line of evidence may tell us more about procedures that restore CS effectiveness than they tell us about what caused the loss of CS effectiveness originally. Omitting the US may indeed restore CS effectiveness, but that does not necessarily imply that the CS originally lost its effectiveness because it reliably predicted that US.

Finally, in opposition to the evidence that a CS loses effectiveness because it predicts a consequence, there is another body of contradictory evidence (Lubos, Alek, \& Arzy, 1975; Lubow, Schnur, \& Rifkin, 1976; Szakmary, 1977). This second body of evidence suggests that one way to minimize the loss of CS effectiveness as a result of CS preexposures is to ensure that the preexposed CS reliably predicts a programmed consequence.

\section{REFERENCES}

ANNAU, Z., \& Kamin, L. J. (1961). The conditioned emotional response as a function of intensity of the US. Journal of Comparative and Physiological Psychology, 54, 428-432.

Berthier, N. E., \& Moore, J. W. (1980). Spatial differential conditioning of the nictitating membrane response in the hippocampectomized rabbit. Physiological Psychology, 25, 667-673.

Dickinson, A., \& Mackintosh, N. J. (1979). Reinforcer specificity in the enhancement of conditioning by posttrial surprise. Journal of $E x$ perimental Psychology: Animal Behavior Processes, 5, 162-177.

Gormezano, I. (1966). Classical conditioning. In J. B. Sidowski (Ed.), Experimental methods and instrumentation in psychology. New York: McGraw-Hill.

Hall, G., \& PeArCe, J. M. (1979). Latent inhibition of a CS during CSUS pairings. Journal of Experimental Psychology: Animal Behavior Processes, 5, 31-42.

HALL, G., \& PEARCE, J. M. (1982). Restoring the associability of a preexposed CS by a surprising event. Quarterly Journal of Experimental Psychology, 34B, 127-140.

IsON, J. R., \& LEONARD, D. W. (1971). Effects of auditory stimuli on the amplitude of the nictitating membrane reflex of the rabbit (Oryctolagus cuniculus). Joumal of Comparative and Physiological Psychology, 75, 157-164.

LUBOW, R. E., ALEK, M., \& ARZy, J. (1975). Behavioral decrement following stimulus preexposure: Effects of number of preexposures, presence of a second stimulus, and interstimulus interval in children and adults. Joumal of Experimental Psychology: Animal Behavior Processes, 1, 178-188.

Lubow, R. E., Schnur, P., \& Rifkin, B. (1976). Latent inhibition and conditioned attention theory. Journal of Experimental Psychology: Animal Behavior Processes, 2, 163-174.

Lubow, R. E., Weiner, I., \& SchnuR, P. (1981). Conditioned attention theory. In G. H. Bower (Ed.), The psychology of learning and motivation (Vol. 15). New York: Academic Press.
Mackintosh, N. J. (1975). A theory of attention: Variations in the associability of stimuli with reinforcement. Psychological Review, 82, 276-298.

Marchant, H. G., III, Mis, F. W., \& Moore, J. W. (1972). Conditioned inhibition of the rabbit's nictitating membrane response. Journal of Experimental Psychology, 95, 408-411.

Marchant, H. G., III, \& Moore, J. W. (1973). Blocking of the rabbit's conditioned nictitating membrane response in Kamin's two-stage paradigm. Journal of Experimental Psychology, 101, 155-158.

MilleNSON, J. R., \& DENT, J. G. (1971). Habituation of conditioned suppression. Quarterly Journal of Experimental Psychology, 23, 126-134.

MOORE, J. W., \& STICKNEY, K. J. (1980). Formation of attentionalassociative networks in real time: Role of the hippocampus and implications for conditioning. Physiological Psychology, 8, 207-217.

Pearce, J. M., \& Hall, G. (1980). A model for Pavlovian learning: Variations in the effectiveness of conditioned but not of unconditioned stimuli. Psychological Review, 87, 532-552.

Rescorla, R. A., \& Wagner, A. R. (1972). A theory of Pavlovian conditioning: Variations in the effectiveness of reinforcement and nonreinforcement. In A. H. Black \& W. F. Prokasy (Eds.), Classical conditioning II. New York: Appleton-Century-Crofts.

Rudy, J. W., \& Cheatle, M. D. (1978). A role for conditioned stimulus duration in toxiphobia conditioning. Journal of Experimental Psychology: Animal Behavior Processes, 4, 399-411.

SCHULL, J. (1979). A conditioned opponent theory of Pavlovian conditioning and habituation. In G. H. Bower (Ed.), The psychology of learning and motivation (Vol. 13). New York: Academic Press.

SOLOMON, P. R., \& MOORE, J. W. (1975). Latent inhibition and stimulus generalization of the classically conditioned nictitating membrane response in rabbits (Oryctolagus cuniculus) following dorsal hippocampal ablation. Journal of Comparative and Physiological Psychology, 89, 1192-1203.

SzAKMARY, G. A. (1977). A note regarding conditioned attention theory. Bulletin of the Psychonomic Society, 9, 142-144.

WaGNER, A. R. (1976). Priming in STM: An information-processing mechanism for self-generated or retrieval-generated depression in performance. In T. J. Tighe \& R. N. Leaton (Eds.), Habituation: Perspectives from child development, animal behavior, and neurophysiology. Hillsdale, NJ: Erlbaum.

WAGNER, A. R. (1978). Expectancies and the priming of STM: In S. H. Hulse, H. Fowler, \& W. K. Honig (Eds.), Cognitive processes in animal behavior. Hillsdale, NJ: Erlbaum.

\section{NOTES}

1. All p values in Experiments 1A-2A are two-tailed.

2. At the request of the editor, we have not formally described all of the experiments that were performed; instead, we mention some of them only briefly in discussing other, more fully described, experiments.

(Manuscript received October 28, 1983; revision accepted for publication July 10, 1984.) 